\title{
Lossy Data Reduction Methods for Haptic Telepresence Systems
}

\author{
Martin Kuschel Philipp Kremer ${ }^{1}$ Sandra Hirche ${ }^{2}$ Martin Buss \\ Institute of Automatic Control Engineering (LSR) \\ Technische Universität München \\ D-80290 Munich, Germany www.lsr.ei.tum.de \\ \{Martin.Kuschel,M.Buss\}@ieee.org
}

\begin{abstract}
Telepresence systems are often deployed in scenarios where communication bandwidth is limited. Consequently, data exchanged between operator and teleoperator has to be reduced. In case of haptic telepresence, data reduction has an influence on the stability of the overall system. This paper provides a step towards a systematic framework for communication data bandwidth reduction in haptic telepresence systems discussing stability for a class of lossy data reduction (LDR) algorithms. Simulation and experimental results validate the efficacy.
\end{abstract}

\section{INTRODUCTION}

Telepresence enables a human operator to perceive and manipulate a remote environment. The human operator handles the human system interface (HSI) thereby commanding the robotic teleoperator (TO) to perform actions in a remote environment. HSI and TO exchange command and sensor feedback signals over a communication network (COM). To achieve holistic remote immersion, multiple modalities of human perception are addressed including the visual, auditory, and haptic sense, see Fig. 1 for an illustration. Applications for telepresence are tele-surgery, tele-assembly and tele-rescue systems, etc. The

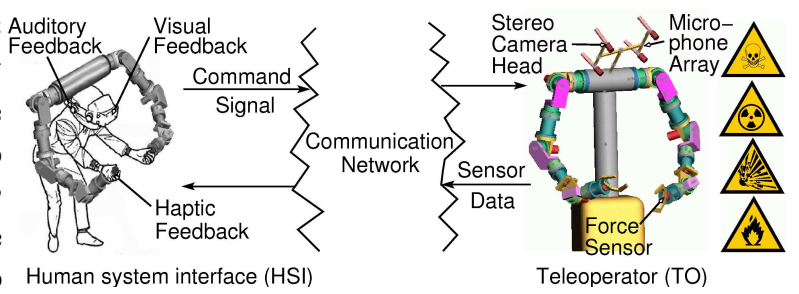

Fig. 1. Multimodal telepresence system.

focus of this paper is on haptic telepresence systems. Haptic perception involves both tactile perception through the skin, like vibrations, and kinesthetic perception of position, force, motion of joints and muscles [1]. By the haptic command and feedback signals energy is bilaterally exchanged between the HSI and the TO. Thereby a global control loop is closed via the communication network (COM). Main objectives in the control system design are stability and transparency; ideal

\footnotetext{
${ }^{1}$ Institute of Robotics and Mechatronics, German Aerospace Center (DLR), Germany; this research has been performed while the author was with the Institute of Automatic Control Engineering (LSR), Technische Universität München.

${ }^{2}$ On leave as JSPS postdoc researcher at Department of Mechanical and Control Engineering, Tokyo Institute of Technology, Japan.
}

transparency means that the operator should feel as if directly being present (immersed) in the remote environment. The key challenges associated with the loop closed over the COM are time delay and limited communication resources. While the time delay problem is well treated in the known literature [2][4], very few researchers in the area of telepresence consider communication resource limitations, e.g. [5]. Quantization to reduce data bandwidth in telepresence has been investigated by [6]-[9], however, stability (passivity) is not investigated. The lossless compression scheme proposed in [6] results in a tradeoff between compression efficiency and time delay required for compression. Differential pulse code modulation (DPCM) together with a fixed rate quantization has been proposed in [8], adaptive DPCM together with Huffman coding has been considered in [9].

In order to provide high immersion with respect to haptic perception, HSI and TO must provide high manipulability [10]. This means, both robots must have several degrees of freedom, resulting in several sensors and several actuators resulting in a large amount of communication data. Generally, the communication bandwidth over common purpose networks, such as the Internet, is limited. Severe communication constraints are induced in space or underwater applications. This motivates investigations towards algorithms for haptic data reduction. In order to design data reduction algorithms it is effective to discard irrelevant (unperceivable) information. The result is a lossy data reduction (LDR) algorithm. Any LDR algorithm also must guarantee stability (passivity) of the overall system. Main contribution of this paper is a classification scheme for a class of LDR algorithms. Sufficient stability (passivity) conditions are derived. Selected LDR algorithms derived from the framework are discussed in detail along with simulation and experimental results.

The remainder of this paper is organized as follows: In Section II a brief background is presented followed evaluation criteria and a classification scheme in Sections III and IV. Simulations and experimental results are presented in Sections V and VI, respectively.

\section{BACKGROUND}

This section introduces basic concepts to stabilize haptic telepresence systems and the formal definition of transparency. 


\section{A. Passivity}

Passivity is an energy-based concept, which provides sufficient stability conditions for interconnected complex systems such as telepresence systems. A passive system does not generate energy. An observable system with zero initially stored energy is passive, if

$$
\int_{0}^{t} P_{i n}(\tau) \mathrm{d} \tau \geq 0, \quad \forall t \geq 0,
$$

where $P_{\text {in }}$ represents the instantaneous power input. If the subsystems of the telepresence system are passive, then overall stability can be ensured. As illustrated in Fig. 2 a telepresence system can be interpreted as a connection of twoports terminated by one-ports at each end. In a velocity-force architecture [2], [3] velocity is commanded to the teleoperator and force is fed back to the human. The power input is

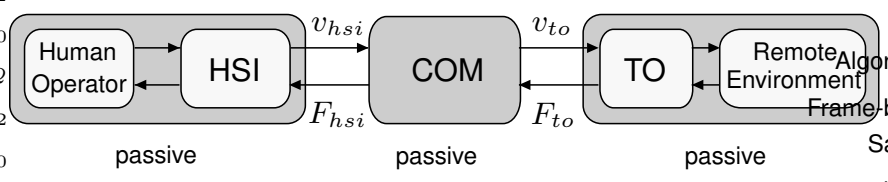

Fig. 2. Haptic telepresence system with velocity-force architecture

then defined as scalar product between effort (force) and flow (velocity) variables, where

$$
P_{\text {in }}=\mathbf{v}^{\mathbf{T}} \mathbf{F}:=v_{h s i} F_{h s i}-v_{t o} F_{t o} .
$$

is the power entering the COM-two-port with $v$ denoting the velocity and $F$ the force; subscripts for HSI and TO. To obtain a passive telepresence system the subsystem consisting of the human operator handling the HSI has to be passive. A trained operator is supposed to assure this. The one-port consisting of the TO working in the remote environment must be passive as well.

A COM-two-port with time delay is not passive. In case of constant communication delay, the active COM can be passivated by the scattering transformation introduced in [2], [3]. The bijective transformation of velocity and force into scattering variables is given by

$$
\begin{array}{cl}
g_{l}= & \frac{b v_{h s i}+F_{h s i}}{\sqrt{2 b}}, \\
g_{r}=\frac{b v_{t o}+F_{t o}}{\sqrt{2 b}}, & h_{l}=\frac{b v_{h s i}-F_{h s i}}{\sqrt{2 b}}, \\
h_{r} & =\frac{b v_{t o}-F_{t o}}{\sqrt{2 b}},
\end{array}
$$

where $g$ describes the incident wave and $h$ the reflected wave. The tuning parameter $b>0$ represents the wave impedance. Using (1), (2) and (3), the energy balance of the COM twoport including the scattering transformation is

$$
\int_{0}^{t} P_{i n}(\tau) \mathrm{d} \tau=\frac{1}{2} \int_{0}^{t}\left(g_{l}^{2}-g_{r}^{2}+h_{r}^{2}-h_{l}^{2}\right) \mathrm{d} \tau .
$$

It is straightforward to show that

$$
\int_{0}^{t}\left(g_{l}^{2}-g_{r}^{2}\right) \mathrm{d} \tau \geq 0 \quad \text { and } \quad \int_{0}^{t}\left(h_{r}^{2}-h_{l}^{2}\right) \mathrm{d} \tau \geq 0, \forall t
$$

represents a sufficient condition for passivity (1). In case of transmission delayed by the time delay $T$ in the forward and the backward path, the power balance (4) can be rewritten as

$$
\int_{0}^{t} P_{i n}(\tau) \mathrm{d} \tau=\frac{1}{2} \int_{t-T}^{t}\left(g_{l}^{2}+h_{r}^{2}\right) \mathrm{d} \tau \geq 0 \quad \forall t,
$$

as $g_{r}(t)=g_{l}(t-T)$ and $h_{l}(t)=h_{r}(t-T)$. The system is passive, i.e stable, for arbitrarily large constant delay.

\section{B. Transparency and Immersion}

A telepresence system is called transparent if the operator is not able to distinguish between direct interaction with an environment and interaction by telepresence. Hence, transparency sets the benchmark of the telepresence system to achieve ideal remote immersion; in [11] the equality of position and force is termed transparency. In this paper the equivalent transparency definition of equal velocities $v$ and forces $F$ at HSI and TO is used as follows

$$
v_{t o} \stackrel{!}{=} v_{h s i} \quad \text { and } \quad F_{h s i} \stackrel{!}{=} F_{t o}
$$

Using this definition of transparency, the integral of the quadratic velocity and force error

$$
I=\int_{0}^{t}\left[\left(v_{h s i}-v_{t o}\right)^{2}+\left(F_{t o}-F_{h s i}\right)^{2}\right] \mathrm{d} \tau .
$$

represents a transparency measure. High cost $I$ indicates a poor level of immersion; zero cost means transparency in terms of (6).

\section{LDR EVALUATION CRITERIA}

LDR algorithms discard or compress information to reduce the amount of data that has to be stored, processed, and transmitted. i.e. the data transmission with LDR is no longer ideal. The LDR acts within the closed control loop. Stability has to be ensured for the haptic telepresence system with LDR. This is the major difference to standard coding algorithms in audio and video applications. Based on the passivity framework as introduced in the previous section a general architecture for haptic telepresence system with LDR is proposed: The bidirectional communication channel is encapsulated by two-ports that perform a passive signal reduction and signal reconstruction as shown in Fig. 3. Time delays either resulting from the data transmission over the network or the LDR algorithm itself are passivated using the scattering transformation. Sufficient conditions for the passivity of LDR and such for the overall stability of the haptic telepresence system are presented in Section IV.

With stability/passivity as a necessary property, transparency and compression ratio are the main performance objectives

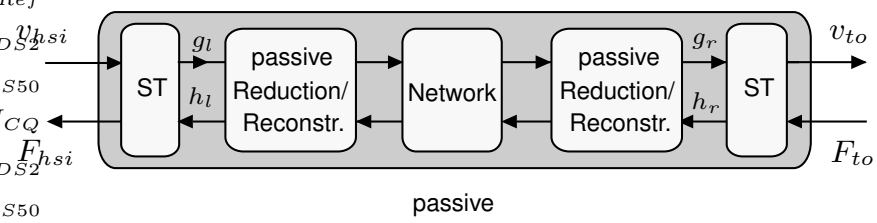

Fig. 3. COM with LDR for haptic telepresence systems. 
in the LDR design. The following criteria are proposed to evaluate passive LDR algorithms:

1. Transparency: A LDR algorithm is called transparent if the human operator does not perceive a difference between an approach without LDR and with LDR. Transparency in this sense is generally difficult to evaluate without human user studies as the human perception characteristics has to be taken into account. Sufficient conditions for transparency (6) cannot be satisfied by any LDR algorithm because at least some data is lost. Accordingly, any of the reviewed transparency measures, such as (7), gives a relative value appropriate for the comparison of LDR algorithms. An absolute statement on transparency remains difficult because of additional time delay and estimation errors introduced by an LDR algorithm.

2. Compression ratio: The compression ratio is defined by the ratio of the amount of original data in the uncompressed lossless case to the amount of reduced data, normalized to the amount of reduced data. The compression ratio is given by $c: 1$. It is desirable to have a high compression ratio indicated by a high number $c>>1$. As it represents a average value a high compression ratio does not implicitely satisfy possible communication bandwidth constraints. Therefore, the maximum data rate, i.e. the minimum instantaneous compression ratio, that can be guaranteed by a LDR algorithm, is of high interest.

In general, the design is subject to a trade-off between achievable transparency and achievable compression ratio, hence the search for the optimal algorithm can be formulated as a multiobjective optimization problem. Accordingly, the optimal LDR algorithm among a set of algorithms

- achieves the maximum transparency level given a certain compression ratio, or

- achieves maximum compression ratio given a certain transparency level.

For a guaranteed minimum instantaneous compression ratio (limited communication bandwidth) the same argument applies.

\section{ClassificATION OF LDR APPROACHES}

The top-down classification scheme proposed in this paper divides the LDR approaches into frame-based and samplebased strategies, see Fig. 4. In a frame-based approach a number of samples is gathered to a frame before the LDR becomes active, i.e. it induces additional time delay into the closed loop. Stabilizing control measures such as the scattering transformation are necessary to guarantee stability. Samplebased methods act on the single sample rather than on a frame without introducing additional time delay.

Frame-based methods are subdivided into frequency-based LDR and interpolative LDR, while the sample-based methods are subdivided into extrapolative and direct methods. These four LDR strategy classes are introduced in the following, and discussed with respect to passivity, transparency, and compression ratio. Passivity conditions are presented. A haptic telepresence system with the constant time delay $T$ in the forward and the backward path is assumed. Consequently, all conditions are specified in scattering variables, cf. (3). The conditions are stated exemplarily for the forward path, however, analogously apply to the backward path. Additional data-preserving coding strategies (Huffman coding, etc.) are not discussed for conciseness but can be readily integrated into the classification scheme as either frame- or sample-based versions.

\section{A. Frame-based Approach: Frequency-Based LDR}

Frequency based LDR algorithms transform the signal from the sender into the frequency domain. A (large) number of samples are gathered into frames, then e.g the Fourier transform is applied, and the data reduction is achieved by transmitting only dominant frequency components of the spectrum. At the receiver site another transformation translates the signal back into the time domain, which is then used for the local control loop.

This approach introduces (possibly large) additional time delay into the telepresence system, where it is known from [12] that already a small increase in time delay significantly impairs transparency; this is the reason why the frequency-based approach is not further elaborated in this paper. The frame length needs to be (quite) large to encode low frequency components of the signals. An advantage of the approach is that a constant data compression ratio can be achieved.

\section{B. Frame-based Approach: Interpolative LDR}

An approach using smaller frame lengths is desirable. The interpolative LDR approximates the incoming signal in the time domain. The algorithm works as follows: $k_{F}$ samples are accumulated to a frame, a signal shaping algorithm, e.g. a spline interpolation, is applied, the resulting parameter vector $\mathbf{p}$ is then transmitted over the network. The receiver reconstructs the signal using the (e.g. spline) parameter vector p. In Fig. 5 the structure of the COM with LDR is illustrated. To comply the passivity conditions (5), every interpolation over the frame length $t_{F}$ has to fulfill

$$
\int_{t_{j}+t_{F}+T}^{t_{j}+2 t_{F}+T} g_{r}(t)^{2} \mathrm{~d} t \leq \int_{t=t_{j}}^{t_{j}+t_{F}} g_{l}(t)^{2} \mathrm{~d} t,
$$

with $t_{j}$ the starting time of frame $j$. That means, the energy of the interpolated signal over the frame length $t_{F}$ has to be less or equal to the energy of the original signal. There are two factors influencing transparency: 1) the induced additional time delay equal to the frame length $t_{F} ; 2$ ) The error resulting

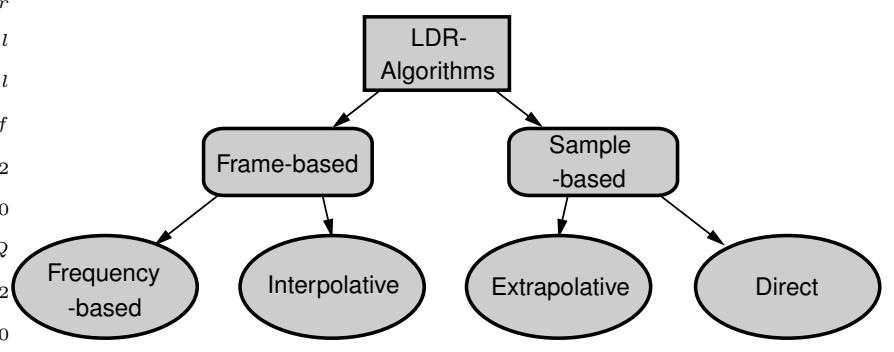

Fig. 4. Classification scheme of haptic LDR algorithms. 


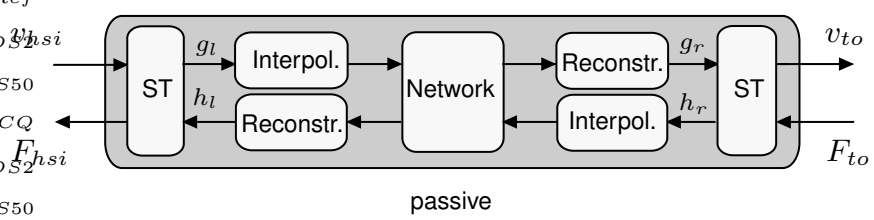

Fig. 5. COM for interpolative LDR

from the reduction of the time series in the frame to a parameter vector $\mathbf{p}$ of a signal shaping algorithm. Given a signal shaping algorithm, the higher the dimension of the parameter vector the lower is the error. Low frame length, i.e. low additional time delay, and high dimension of the parameter vector will lead to good transparency. However, a high compression ratio can only be achieved for a high frame length and a low dimensional parameter vector. The compression ratio per frame is $c: 1$ with

$$
c=\frac{k_{F}}{\operatorname{dim}(\mathbf{p})},
$$

and $k_{F}$ the number of samples in one frame. The data rate is constant, hence the instananeous compression ratio is equal to the compression ratio on average. Communication bandwidth constraints can be satisfied. The trade-off between transparency and compression ratio is adjustable according to the requirements.

\section{Sample-based Approach: Extrapolative LDR}

The extrapolative strategy deploys an estimation of the next $k_{e s}$ samples, called estimation horizon, at the sender side to achieve a reduction. An extrapolation algorithm estimates the future signal parameters are transmitted within the allowable passivity (energy) limits. No additional time delay is induced. The structure is the same as shown in Fig. 5. Instead of an interpolation an extrapolation is performed and the resulting parameter vector $\mathbf{p}$ is transmitted over the network. Every $k_{e h}$ samples an estimation of the next $k_{e h}$ samples is conducted. To assure a passive estimation according to (5) the energy of the estimated samples has to be smaller than the difference between the unshaped signal energy and the energy used to estimate the past samples. Formally, the following passivity constraint has to hold

$$
\int_{0}^{t_{j}}\left(g_{l}^{2}-g_{r}^{2}\right) \mathrm{d} t \geq \int_{t_{j}+T}^{t_{j}+t_{e h}+T} g_{r}^{2} \mathrm{~d} t
$$

with $t_{j}$ the time when a new estimation is performed and $t_{e s}$ the duration of the estimation horizon. Any estimation algorithm can be used as long as (10) is satisfied.

The advantages of the approach are similar to the advantages of the interpolative LDR. However, in place of an approximation this strategy uses an estimation and in place of frames it uses estimation series. The compression ratio is similar to (9) replacing $k_{F}$ by $k_{e s}$.

\section{Sample-based Approach: Direct LDR}

Within the direct LDR strategy, the reduction scheme is performed on each sample directly. An example for a direct
LDR strategy is coarser quantization. The structure is again shown in Fig. 5. Instead of an interpolation the direct scheme is deployed. The passivity condition (5) is fulfilled by assuring that the absolute value of the incoming sample $g_{r}(t+T)$ has to be decreased or left unchanged compared to the associated sent sample $g_{l}(t)$

$$
\left|g_{l}(t)\right| \geq\left|g_{r}(t+T)\right| .
$$

The main advantage of the approach is due to its direct character: No delays are induced and the remote immersion is left unchanged beside the additional quantization noise. Furthermore, the passivity constraint (11) is straightforward and easy to fulfill. The average data reduction as well as the upper bandwidth can be adjusted by the chosen resolution. A related approach is deadband control as proposed in [5], which results in non-uniform sampling and is therefore somewhat different. Approaches using (adaptive) differential pulse code modulation are also direct LDR algorithms, see [8], [9].

\section{E. Summary: LDR classification}

The interpolative, extrapolative, and direct strategy form a classification framework for many possible LDR algorithms. The conditions (8), (10), (11) assure passivity of algorithms of the specific class.

\section{Simulations}

This section provides simulations of three algorithms. Each of the algorithms represents one of the explained classes of algorithms: interpolative LDR, extrapolative LDR and direct LDR. The strategies are applied to the scattering variables. In order to evaluate the influence of the LDR algorithm only, the time delay is set to $T=0$. The remote environment is modeled by an impedance with parallel spring-damper behavior with the transfer function $1 /(s+1)$. The human operator exerts a velocity step. Its reaction is modeled by an admittance with serial spring-damper dynamics with the transfer function $s /(s+0.5)$. The dynamics of HSI and TO are assumed to be negligible, they are modeled as ideal transducers with unity transfer functions. The sample frequency is $1000 \mathrm{~Hz}$. The velocity and force responses of the HSI and the TO are presented to demonstrate the effect of the different algorithms. The transparency criterion (7) is computed over the simulation horizon of $5 \mathrm{~s}$ and normalized to the highest value. For the standard approach without LDR, the transparency criterion is $I_{R e f}=0$ as expected; naturally, the compression ratio is $1: 1$.

\section{A. Interpolative LDR: Passive Interpolative Down Sampling}

For the interpolative LDR a passive interpolative down sampling (iDS) is applied, the parameter vector $\mathbf{p}$ contains the mean value over the frame length. It is straightforward to show that this strategy satisfies the passivity condition (8). The results for the interpolative LDR for frame-lengths of $k_{F}=2$ and $k_{F}=50$ are shown in Fig. 6 and Fig. 7, respectively. The transparency is decreased for the higher frame-length resulting from additional time delay and interpolation error: 
higher frequencies are filtered out. The transparency criterion values are $I_{i D S 2}=0.05$ and $I_{i D S 50}=1$, respectively. As the dimension of the transmitted parameter vector is $\operatorname{dim}(\mathbf{p})=1$ in both cases, a compression ratio (9) of $2: 1$ and $50: 1$ (equivalent to a data reduction of $50 \%$ and $98 \%$ ), respectively, is achieved.
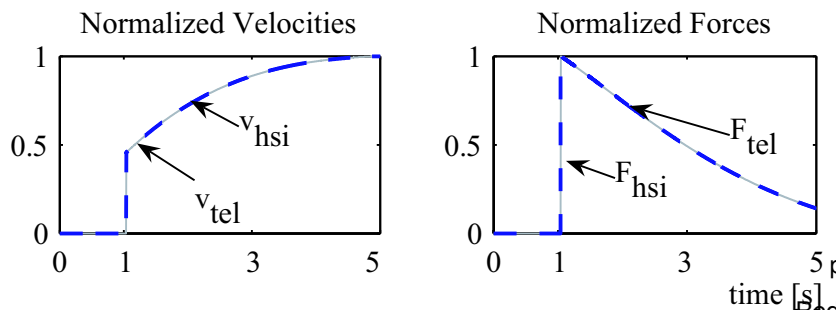

Fig. 6. Interpolative down sampling with frame length $k_{F}=2$ achieves $2: 1$ compression ratio and high immersion.
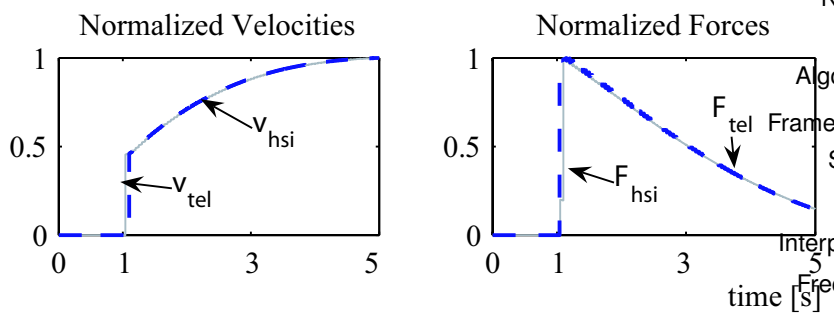

Fig. 7. Interpolative down sampling with frame length $k_{F}=50$ achieves $50: 1$ compression ratio. Higher frequencies are filtered out.
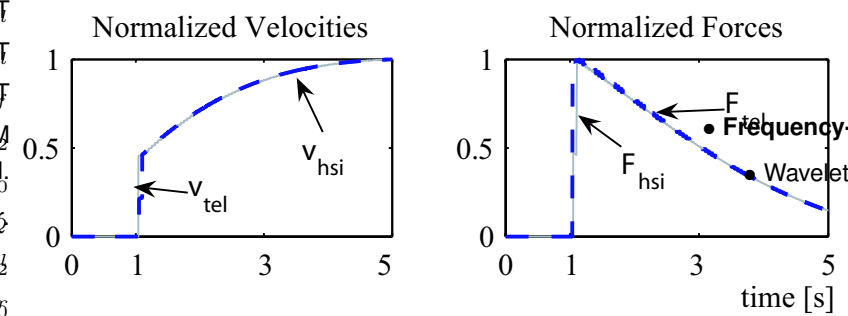

Fig. 8. Extrapolative down sampling with frame length $k_{e s}=50$ achieves $50: 1$ compression ratio.
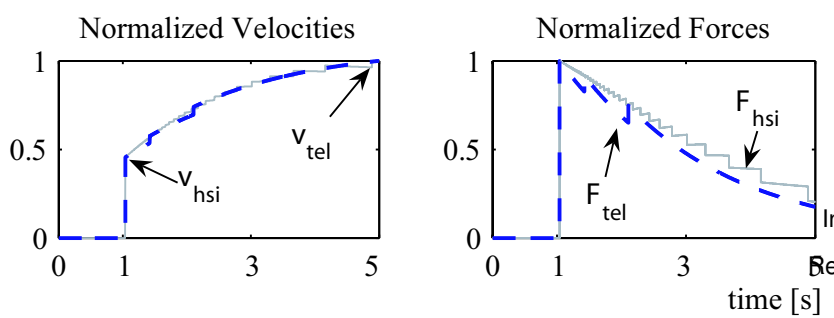

Fig. 9. Coarser quantization achieves $2: 1$ compression ratio. Noise decreases transparency.

\section{B. Extrapolative LDR: Passive Extrapolative Down Sampling}

Similar to the interpolative LDR, for the extrapolative LDR a passive extrapolative down sampling (eDS) is applied. Here again a single value is transmitted parameter vector: $\operatorname{dim}(\mathbf{p})=1$. This value is either the most recent value measured, if (10) is satisfied or a value computed such that (10) is satisfied. This strategy is passive as straightforward to show. The result for an estimation horizon of $k_{e s}=50$ is depicted in Fig. 8, the result for $k_{e s}=2$ is not explicitly shown as it is very similar to Fig. 6. The transparency decreases with higher estimation horizon as observable from the transparency criterion values $I_{e D S 2}=0.03$ and $I_{e D S 50}=0.61$. In contrast to the interpolative LDR no additional delay is introduced, the decreased transparency results from the estimation error only. As in the previous case, the compression ratio is $2: 1$ and $50: 1$, respectively.

\section{Direct LDR: Coarser Quantization}

As a direct LDR scheme a coarser quantization (CQ) is implemented: the standard quantization of $16 \mathrm{Bit}$ is reduced to 8 Bit quantization. The passivity condition (11) is satisfied by transmitting the next lower (in an absolute sense, i.e. closer to zero) quantization value. The coarser quantization of $8 \mathrm{Bit}$, as shown in Fig. 9, introduces a quantization noise deteriorating transparency. The immersion value is $I_{C Q}=0.34$ while the compression ratio is $2: 1$.

The trade-off between transparency and compression ratio, stated in Section III is clearly observable. Comparing the transparency for the compression ratio of $2: 1$ the passive extrapolative downsampling approach is superior over the other two approaches.

\section{EXPERIMENTS AND EVALUATION}

An experimental evaluation of the simulated algorithms is performed using a two degree-of-freedom (2-DoF) haptic telepresence system. The experimental set up is shown in Fig. 10. It consists of two identical SCARA-robots with two degrees of freedom connected to a PC. The link angles are measured by an incremental encoder, the torque applied to each link by strain gauges. The sensor data are processed in the PC running unter RT/Linux. All control algorithms (HSI force control, teleoperator velocity control in the joint space) including the LDR algorithms are implemented as Matlab/Simulink models with realtime code generated from them. The control loops operate at a sampling rate of $1000 \mathrm{~Hz}$ representing the communication rate without data reduction algorithms. The time delay is set to $T=0$ in order to evaluate the influence of the LDR algorithms only. For each algorithm 10 runs are performed where the human operator is moving in free space in the time interval $0 . .5 \mathrm{~s}$, pushes into a damping environment (foam) in the time interval $5 . .10 \mathrm{~s}$, and finally pushes twice against a stiff surface in the time interval $10 . .15 \mathrm{~s}$. In Fig. 11 the velocity and force measured at the inner links of HSI and TO are depicted for the reference (no LDR) experiment for an example run. Example velocity and force responses for interpolative down sampling (iDS) with $k_{F}=2$,

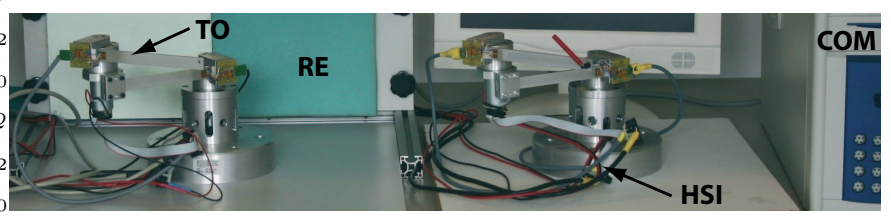

Fig. 10. Experimental 2-DoF haptic telepresence system. 
extrapolative down sampling (eDS) with $k_{e s}=50$ and a coarser quantization of 8,Bit (CQ) are shown in Fig. 12, Fig. 13 and Fig. 14, respectively. The mean values and the standard deviation over the performed 10 runs of the transparency criterion (7) are presented in Fig. 15. As in the simulation among the LDR algorithms, the extrapolative down sampling approach performs best comparing the results for a compression ratio of $2: 1$ (iDS2, eDS2, CQ). Its transparency is comparable to the reference experiment. For an absolute statement on the transparency of these LDR algrithms as defined in Section III, human user studies are necessary being subject of future research.
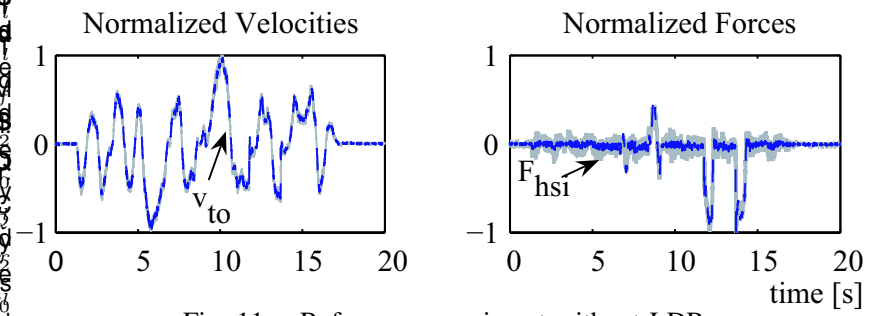

Fig. 11. Reference experiment without LDR.
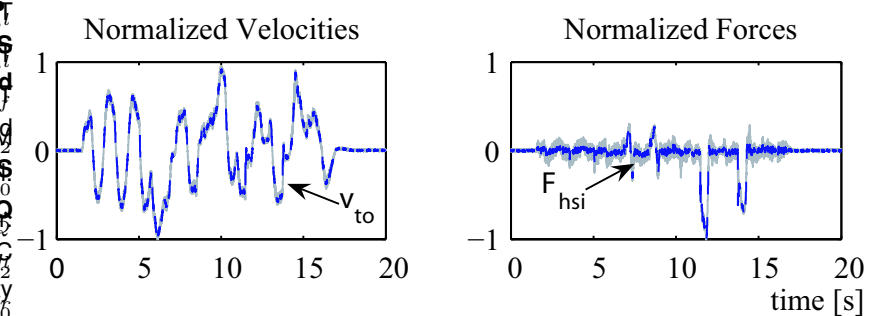

Fig. 12. Interpolative down sampling with frame length $k_{F}=2$ shows good transparency.
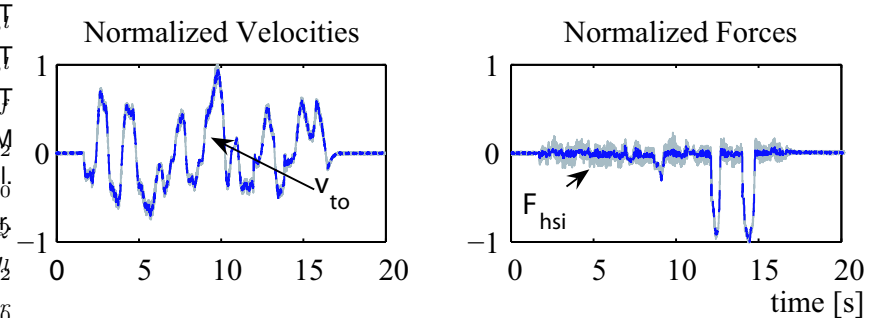

Fig. 13. Extrapolative down sampling with frame length $k_{e s}=50$ : Transparency deteriorated.
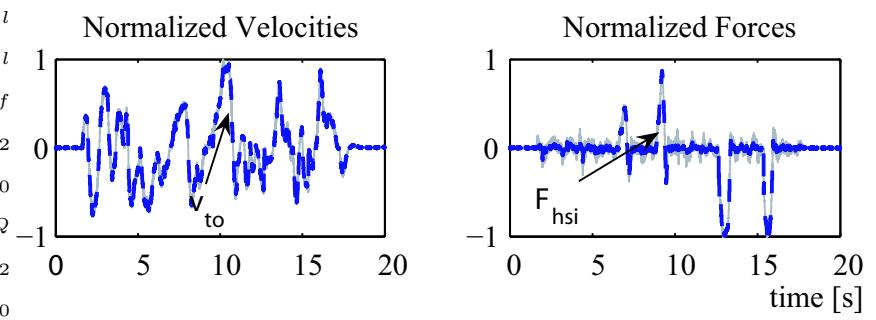

Fig. 14. Coarser quantization of 8Bit: Transparency deteriorated.

\section{CONCLUSION}

This paper proposes a systematic classification framework for lossy data reduction (LDR) algorithms in haptic telepresence systems. Two groups of sample-based and framebased approaches include direct, interpolative, extrapolative,

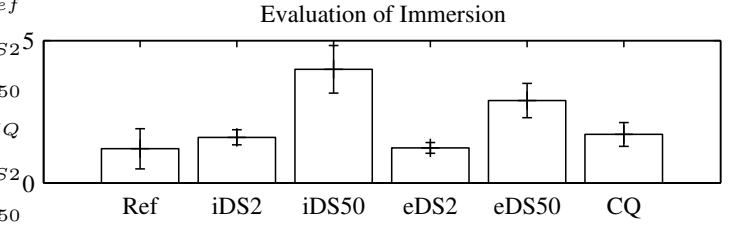

Fig. 15. Transparency comparison: Mean value and standard deviation over 10 runs for the reference approach (no LDR) and considered LDR algorithms.

and frequency-based algorithms. Sufficient passivity (stability) conditions are stated as a design guideline for LDR algorithms applicable to haptic telepresence systems with time delay. Simulation and experimental results validate selected LDR algorithms in their performance in terms of transparency and data compression ratio.

Future investigations are to analyze issues of human perception such as LDR algorithm design using psychophysical insight, e.g. just-noticable-difference thresholds for motion and forces.

\section{ACKNOWLEDGMENT}

This work is supported in part by the German Research Foundation (DFG) within the collaborative research center SFB453, http: / / www. sfb453.de.

\section{REFERENCES}

[1] E. Goldstein, Sensation and Perception. 6th ed., 2002

[2] R. Anderson and M. Spong, "Bilateral Control of Teleoperators with Time Delay," IEEE Transactions on Automatic Control, vol. 34, no. 5, pp. 494-501, 1989.

[3] G. Niemeyer and J.-J. Slotine, "Stable Adaptive Teleoperation," IEEE Journal of Oceanic Engineering, vol. 16, pp. 152-162, January 1991.

[4] Y. Yokokohji, T. Imaida, and T. Yoshikawa, "Bilateral Control with Energy Balance Monitoring under Time-Varying Communication Delay," in Proceedings of the IEEE International Conference on Robotics and Automation, (San Francisco (CA), US), pp. 2684-2689, 2000.

[5] S. Hirche, P. Hinterseer, E. Steinbach, and M. Buss, "Network Traffic Reduction in Haptic Telepresence Systems by Deadband Control," in Proceedings IFAC World Congress, International Federation of Automatic Control, (Prague, Czech Republic), 2005.

[6] C. Shahabi, A. Ortega, and M. R. Kolahdouzan, "A comparison of different haptic compression techniques," in in Proceedings of the International Conference on Multimedia and Expo (ICME), (Lausanne, Switzerland), pp. 657-660, 2002.

[7] K. Hikichi, H. Morino, I. Fukuda, S. Matsumoto, Y. Yasuda, I. Arimoto, M. Iijima, and K. Sezaki, "Architecture of haptics communication system for adaptation to network environments," in in Proceedings of the International Conference on Multimedia and Expo (ICME), (Tokyo, Japan), pp. 744-747, 2001.

[8] C. W. Borst, "Predictive coding for efficient host-device communication in a pneumatic force-feedback display," in in Proceedings of the First Joint Eurohaptics Conference and Symposium on Haptic Interfaces for Virtual Environment and Teleoperator Systems, (Pisa, Italy), pp. 596599, 2005.

[9] A. Ortega and Y. Liu, "Lossy compression of haptic data," in Touch in Virtual Environments: Haptics and the Design of Interactive Systems (G. S. M. McLaughlin, J. Hespanha, ed.), pp. 119-136, Prentice Hall, 2002.

[10] T. Sheridan, "Musings on Telepresence and Virtual Telepresence," Presence, vol. 1, pp. pp.120-125, 1992.

[11] Y. Yokokohji and T. Yoshikawa, "Bilateral Control of Master-Slave Manipulators for Ideal Kinesthetic Coupling Formulation and Experiment," IEEE Transactions on Robotics and Automation, vol. 10, no. 5, pp. 605619, 1994.

[12] S. Hirche and M. Buss, "Insights on Human Adapted Control of Networked Telepresence and Teleaction systems," International Journal on Human-friendly Welfare Robotic Systems, to appear 2006. 